\title{
Image investigation using higher moment statistics and edge detection for recognizing abnormal skin
}

\author{
Afdhal Afdhal, Zulfikar Zulfikar, Zulhelmi Zulhelmi, Teuku Yuliar Arif \\ Department of Electrical and Computer Engineering, Universitas Syiah Kuala, Indonesia
}

\begin{tabular}{|c|c|}
\hline Article Info & ABSTRACT \\
\hline Article history: & This paper discusses the recognition of skin abnormalities by investigating \\
\hline Received Jul 29, 2018 & $\begin{array}{l}\text { the images using high-level statistics and edge detection. Six images of skin } \\
\text { diseases were analyzed using several statistical parameters, including }\end{array}$ \\
\hline Revised Dec 1, 2018 & high-level moments such as skewness and kurtosis. In comparison, \\
\hline Accepted Sep 26, 2019 & $\begin{array}{l}\text { some images from other categories such as animal, architecture, art, vehicle, } \\
\text { food, people, and scenery have been analyzed as well. The results were }\end{array}$ \\
\hline Keywords: & $\begin{array}{l}\text { compared to skin disease images. It is expected that the general pattern of } \\
\text { statistical parameters can distinguish skin images against images from other }\end{array}$ \\
\hline Edge detection & categories. MATLAB is used as a medium to calculate the values of statistical \\
\hline Image processing & Meanwhile, the standard deviation is the smallest compared to other \\
\hline Kurtosis & categorical images. Almost all the analyzed images close to symmetry. \\
\hline Skewness & Nearly all images category are distributed more leaning to the left, except for \\
\hline Skin image & $\begin{array}{l}\text { the images of the art category, which is slightly more leaning to the right. } \\
\text { Moreover, the edge detection process has been done using the Sobel algorithm. } \\
\text { The result, however, cannot clearly distinguish a skin's abnormality. } \\
\text { This difficulty is because of the lack of accuracy in selection } \\
\text { the intensity. }\end{array}$ \\
\hline
\end{tabular}

This is an open access article under the CC BY-SA license.

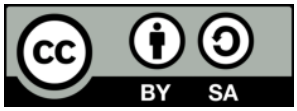

\section{Corresponding Author:}

Afdhal Afdhal,

Department of Electrical and Computer Engineering,

Universitas Syiah Kuala, Jl. Syech Abdul Rauf No. 7,

Darussalam, Banda Aceh, 23111, Indonesia .

Email: afdhal@unsyiah.ac.id

\section{INTRODUCTION}

The increasing use of mobile electronic devices makes it easy for people to interact with each other. The increase also happens in remote areas, as more and more telecommunications devices are available. Besides, interacting with the virtual world has become a trend everywhere. There are no questions that cannot be answered at this time. Someone will quickly find an answer from the internet [1]. The phenomena are also supported by the increasing number of available information in cyberspace [2, 3]. Most people will write down the knowledge they know on the online site so that anyone can read it. In the field of health, more and more websites are also available that provide information on how to keep healthy and also how to treat it. However, skin disease is a disease that is often ignored by people, especially those who live in remote areas [4-7]. Often, new patients come to health workers when the disease becomes severe [8,9]. So we need another solution to this problem. One way to overcome these problems is by the rapid analysis of skin disea ses through mobile phones. The method is in line with the increasing number of mobile phone users in remote areas.

This study aims to find ways to analyze skin diseases through images. The analyzed image is the result of photos of diseased or unhealthy skin. Typical features of the images will be analyzed by 
calculating several sta tistical pa ra meters such as mean, median, standard deviation, and other higher moments. Then further a nalysis will also be done through the edge detection of normal skin image and skin disea se ima ge. Both studies are performed on MATLAB using the Sobel algorithm.

In this paper, some fundamental theories of imaging in MATLAB, and its processing are covered in the next section. Section III provides a detailed comparison between skin disease images and other image categories. Section IV provides the process of edge detection of the normal skin image and abnormal skin images. Finally, the summary of the presented work is presented in section V.

\section{BACKGROUND THEORY}

\subsection{Higher moment statistic}

Skewness and kurtosis are two of the statistics of the higher moments. Skewness is an asymmetry of a probability distribution of its mean $[10,11]$. A qualitative understanding of the skew of distribution is complicated. Skewness can mean positive, negative, or completely undefined. In the unimodal distribution, the negative skewness value shows the tail from the left side of the probability density function (pdf) longer or thicker than the other side. Conversely, if skewness is positive, then the right side is longer or thicker [12]. Figure 1 shows an example of distribution with positive skewness. While in a discrete or multimodal distribution, it is challenging to interpret the meaning of the slope. However, the important thing is that the slope is not unbound to the mean and median, so in some cases, the data can be changed so similar to the normaldistribution [13].

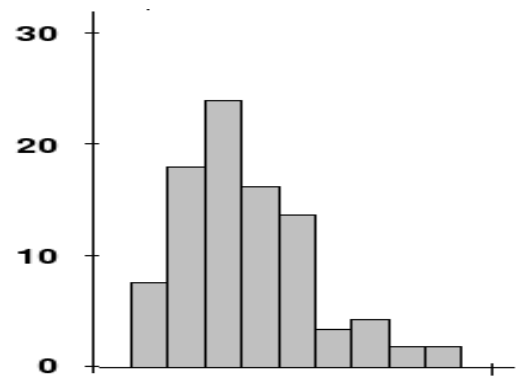

Figure 1. Example distribution with non-zero (positive) skewness

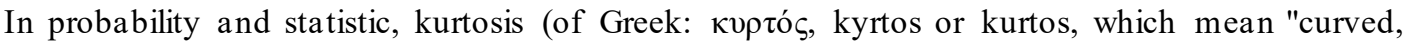
arching") is a measure of the "tailedness" of the probability distribution of a real-valued random variable [14]. Likewise, to the concept of skewness, kurtosis is a parameter often used to explain the shape of the distribution. There are different ways of skewness quantifying for a theoretical distribution and corresponding ways of viewing it from a sample from a population. In practice, there are various explanations of kurtosis, and of how particular measures have to be translated. According to Karl Pearson, kurtosis measurements are based on scale transcription from the fourth moment of the data or population. So according to these measurements, kurtosis is related to the tail of the distribution (not the peak) [4]. Therefore, there is often an error in measuring kurtosis because it takes into account its peak. So if kurtosis is of high value, it means that there is an extreme aberration.

In the Normal distribution, the value of kurtosis involving a variate is 3. Any distribution whose kurtosis value is less than 3 is defined as platykurtic, but not necessarily the distribution is flat-topped $[15,16]$. The uniform distribution is one example of platykurtic distribution because it has no tail. Whereas if a distribution has a value of kurtosis greater than 3 is defined as leptokurtic. Laplac e distribution is one example, where its tail is not symmetrical and tends to be near zero (more gradual than Gaussian distribution). In practice, people often use Pearson's improvement model, i.e., minus 3 kurtosis, to distinguish it from the Normal distribution [17]. Several authors use "kurtosis" by themselves to lead to the excess kurtosis. However, in general, there is no precise meaning of kurtosis that reflects the description of distribution.

\subsection{Edge detection}

The Sobel edge detectors use the masks in Figure 2 to approximate digitally the first derivatives $G x$ and $G y$. In another word, the gradient at the center point in neighborhoods is computed as follows (1) by the Sobel detectors [18, 19]. 


$$
\begin{aligned}
& g=\left[G_{x}^{2}+G_{y}^{2}\right]^{\frac{1}{2}}=\left\{\left[\left(z_{7}+2 z_{8}+z_{9}\right)-\left(z_{1}+2 z_{2}+z_{3}\right)\right]^{2}\right. \\
& \left.+\left[\left(z_{3}+2 z_{6}+z_{9}\right)-\left(z_{1}+2 z_{4}+z_{7}\right)\right]^{2}\right\}^{1 / 2}
\end{aligned}
$$

Then, we saw that a pixel at location $(x, y)$ is an edge pixel if $g \geq T$ at that location. Where $T$ is a specified threshold. Sobel detection technique can be implemented by filtering an image (using imfilter) with the left masking, filtering again with the other mask, squaring the pixels values of each filtered image, adding the two results, and computing their square root.

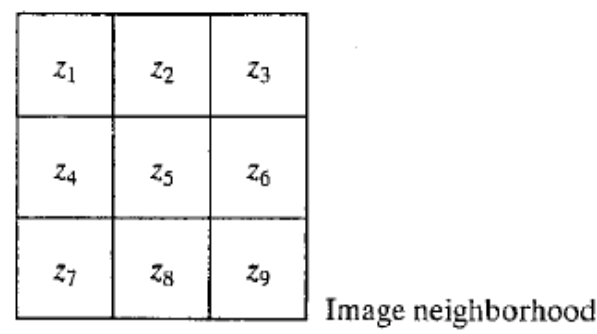

\begin{tabular}{|c|c|c|}
\hline-1 & -2 & -1 \\
\hline 0 & 0 & 0 \\
\hline 1 & 2 & 1 \\
\hline
\end{tabular}

$\left(z_{1}+2 z_{2}+z_{3}\right)$

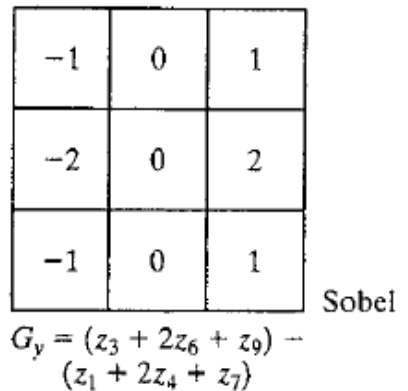

Figure 2. Edge detector masks and the first-order derivatives they implement

\section{STATISTIC COMPARISON OF SKIN IMAGE AGAINST OTHER IMAGES}

Each image has unique color features that distinguish it from others [20]. Likewise, the image of human skin has different color parameters with other images. We can quickly recognize which image of the skin and which is not just by looking at the pictures. However, in digital image processing, the difference between an image and another image is based on the color characteristics [21]. Therefore, we must find the distinctive features of an image so that the processing can be recognized.

In general, a group of images (not individual images) has distinctive features that distingu ish them from others. Therefore, it is necessary to group the pictures into several categories. To obtain the characteristics of the skin images, it is essential to a nalyze and trace some parameters that distinguish them from other groups of images. Here are some categories of images that will be studied in this paper, such as animal, architecture, art, vehicle, food, people, and scenery. The grouping of these images is only based on general observations, but there may be overlap with images that can fit into more than one category.

Figure 3 shows some images of randomly selected skin diseases, ignoring the types of diseases to represent different kinds of images. The pictures have been reduced, adjusted, and uninformed to $100 \times 100$ pixels. The process of clip images to eliminate the effect of various background colors. Selection of these sizes to facilitate the analysis process, although the actual size of the image is less enjoyable when viewed with the eye.

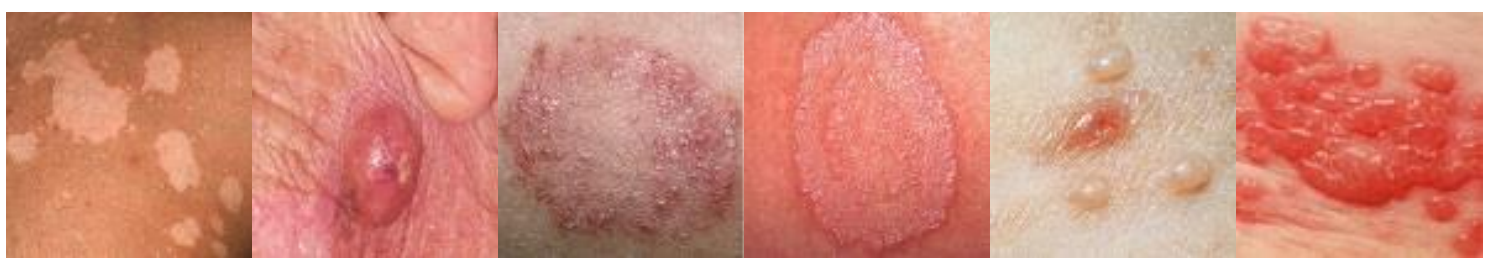

Figure 3. Six images for representing various skin disea ses 
The images will be analyzed statistically using MATLAB. Therefore, all of these images will be captured and represented by a 3D matrix in RGB color space. In the space, the image is represented by the number in an 8-bit integer format or its value from 0 to 255. Some statistical parameters already extracted, such as minimum value, maximum value, the range of values, mean, median, and standard deviation [22]. An additionalparameter, such as skewness and kurtosis, will be investigated as well. Table 1 shows the results of the analysis of some statistical parameters on the six skin images shown in Figure 3.

The data that are shown in Table 1 is a 3 -dimensional matrix, which is a representation of the RGB color model. The matrices contain numbers whose range approach a maximum of 255. Therefore, the numbers in the matrix do not show the typical properties of the specificness of the skin image. The mean and median values which averaged in the middle indicate that the distribution is quite evenly distributed throughout the range of values. It can also be seen that the lowest standard deviation score is 28.32 and the highest is 61.93. Meanwhile, the maximum skewness value is about 0.42 with a minimum value of about -1.03 , indicating all the distributed data is more leaning to the left except skin5. The level of transient distribution around the most frequent values is shown by the parameters of kurtosis. The skin 5 image has a more pointed distribution of the normal distribution because its kurtosis value is greater than 3 , while other skin disease images have a flatter(platykurtic) peak.

Table 1. Properties of representationalmatrices of images of skin disease

\begin{tabular}{|c|c|c|c|c|c|c|c|c|}
\hline & Min & Max & Range & Mean & Median & Standard Deviation & Skewness & Kurtosis \\
\hline skin 1 & 24 & 255 & 231 & 140.62 & 131 & 50.44 & 0.3336 & 1.9603 \\
\hline $\operatorname{skin} 2$ & 33 & 255 & 222 & 158.44 & 147 & 48.93 & 0.2549 & 1.8813 \\
\hline $\operatorname{skin} 3$ & 23 & 255 & 232 & 144.86 & 144 & 42.52 & 0.0755 & 2.1704 \\
\hline $\operatorname{skin} 4$ & 47 & 255 & 208 & 152.85 & 136 & 54.67 & 0.4219 & 1.8398 \\
\hline skin 5 & 31 & 255 & 224 & 188.95 & 193 & 28.32 & -1.0340 & 4.7845 \\
\hline skin 6 & 19 & 255 & 236 & 146.51 & 142 & 61.93 & 0.0130 & 1.6277 \\
\hline
\end{tabular}

Figures 4-10 show some drawings of the randomly selected animal, architecture, art, vehicle, food, people, and scenery categories. Those figures were captured in the Pinterest and Instagram websites [23, 24]. The images have been reduced, adjusted, and uninformed to $100 \times 100$ pixels. The process of clip images to minimize the effect of various background colors. Selection of these sizes to facilitate the analysis process, although the actual size of the image is not very good when viewed with the eye.

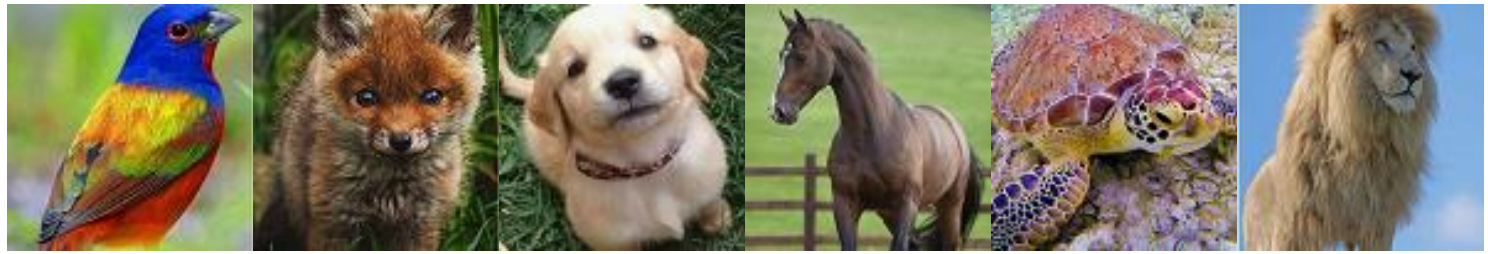

Figure 4. Six images for representing various a nimal

The data shown in Table 2 is a 3-dimensional matrix, which is a representation of the RGB color model. The matrices contain numbers whose range approach almost maximum of 255. Therefore, the numbers in the matrix do not show the typical properties of the specificness of the animalfigure. Regarding animalcategory, the mean and median values which are on a verage slightly left co mpared with the middle of the range indicate that the distribution is quite evenly distributed across the entire range of values. It can also be seen that the lowest standard deviation score is 46.98, and the highest is 65.10. Meanwhile, the maximum skewness value is about 0.86 with a minimum value of about -0.39 , indicating some of the distributed data is more leaning to the left, and the other part is more leaning to the right. The animal2 image has a distribution similar to the normal distribution because the value of the kurtosis is about 3 , while the other animalimages have a flatter peak (platykurtic).

Figure 5 shows some images of randomly selected architecture categories without taking into account all types of images to represent the architecture category [23]. In the architecture category shown in Table 2, the matrices contain numbers whose range maximum of 255 except the figure of architechture6. Therefore, the numbers in the matrix do not show the typicalproperties of the specificness of the architecture view. Moreover, the different mean and median values that tend to be inconsistent indicates that the spread 
is also quite variable across the entire range of values. It can also be seen that the lowest standard deviation

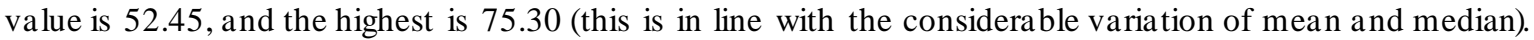
Meanwhile, the maximum skewness value is about 0.85 , with a minimum value of about -1 , indicating almost some distributed data is more leaning to the left except architechture 4 and architechture 5 . The architecture 3 and architechture 5 images have a distribution similar to the normal distribution because the kurtosis value is about 3 , while the other architecture images have a flatter (platykurtic) peak.

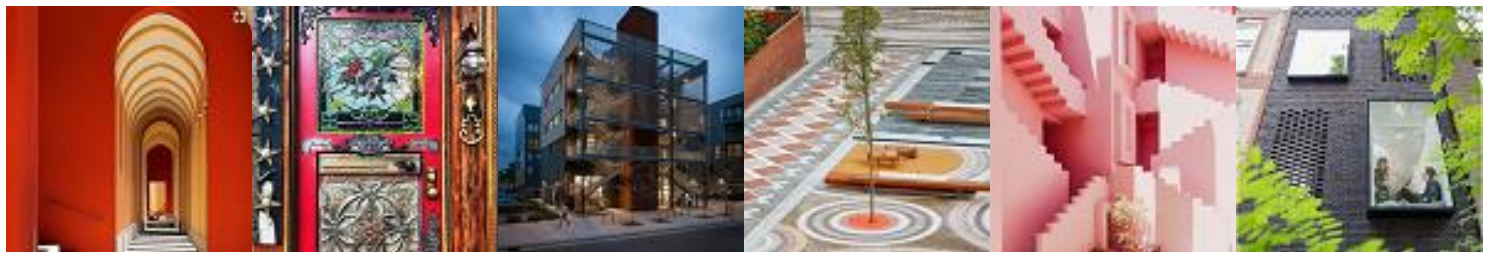

Figure 5. Six images for representing various architecture

Figure 6 shows some drawings of randomly chosen art categories without taking into account all types of images to represent the category [23]. Regarding Art group data showing in Table 2, the matrices contain maximum numbers (255) except the figure of art2. The mean and median values that vary considerably and tend to be very inconsistent indicates that the spread is also quite variable across the entire range of values. It can also be seen that the lowest standard deviation score is 33.59, and the highest is 87.62 (this is in line with the considerable variation of mean and median). Meanwhile, the maximum skewness value is about 1.27, with a minimum value of about -1.38 , indicating that almost some distributed data is more leaning to the left except $\operatorname{art} 4$ and $\operatorname{art} 6$. Images of $\operatorname{art} 2$, art4, and $\operatorname{art} 6$ have more ruth distributions than normal distributions because the kurtosis value is greater than 3 , while other art images have flatter peaks (platykurtic).
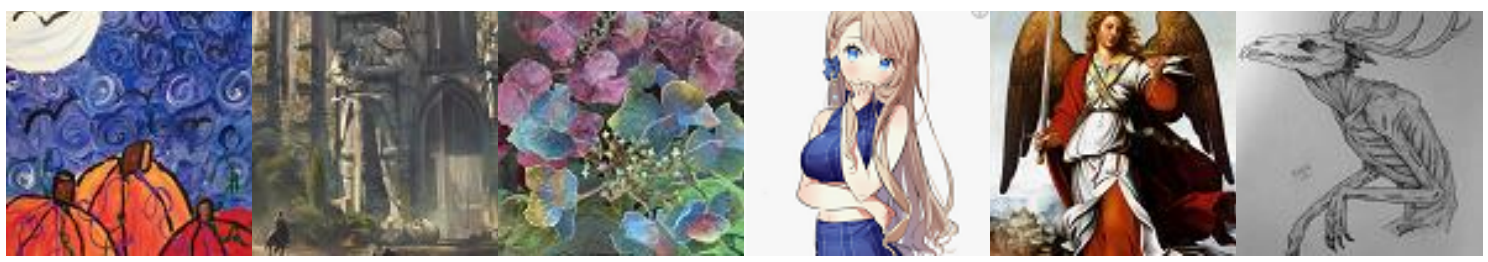

Figure 6. Six images for representing various art

Figure 7 shows some images of randomly chosen vehicle categories without taking into account all types of images to represent the group [23]. The data shown in Table 2 indicate that the range is a maximum of 255. Thus, the numbers in the matrix do not show the typical properties of the specificness of the vehicle view. Mean and median of vehicle images are varied considerably and tend to be inconsistent indicates that the spread is also quite variable across the entire range of values. It can be seen that the lowest standard deviation value is 58.55 , and the highest is 90.56 (this is in line with the significant variations of the mean and median). The maximum skewness value is about 0.95 with a minimum value of about -0.12 , indicating some distributed data is more leaning to the left, and some more data is leaning to the right. Figure vehicle6 has a more ruth distribution than the normal distribution because the kurtosis value is greater than 3 , while the othervehicle images have a flatter peak (platykurtic).

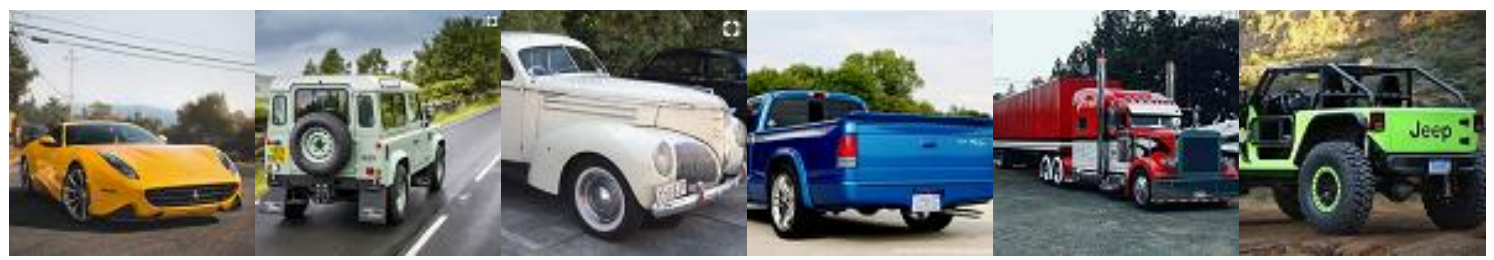

Figure 7. Six images for representing the various vehicle 
Figure 8 shows some images of randomly selected food categories without taking into account a ll types of images to represent the class [23]. The data listed in Table 2 (part of food), which their matrices contain numbers whose range is a maximum of 255. Again, the numbers in the matrix do not show the typical properties of the specificness of the food view. Mean and median are varied considerably and tend to be inconsistent indicates that the spread is also quite variable across the entire range of values. The lowest standard deviation value is 58.26, and the highest is 75.24 (this is in line with the less significant variations of the mean and median). Meanwhile, the maximum skewness value is about 0.72 with a minimum value of about -0.18 , indicating some of the data is distributed more leaning to the left, and some more data is leaning to the right. The level of transient distribution around the most frequent values is shown by the parameters of kurtosis. All food category images have a flatter (platykurtic) distribution than the normaldistribution.

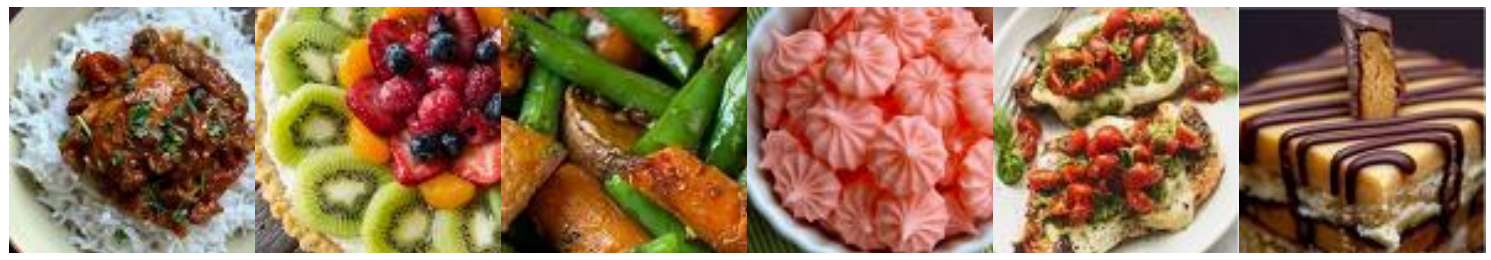

Figure 8. Six images for representing various food

Figure 9 shows some pictures of randomly selected people categories without taking into account all types of images to represent the group. Those figures were captured in the Pinterest and Instagram websites [23, 24]. Based on data from people figures in Table 2, their matrices contain numbers whose maximum range is 255 . Thus, the numbers in the matrix do not indicate the typical nature of the peculiarities of the public view. From mean and median values that vary considerably and tend to be inconsistent, the spread is also quite variable across the range. It can also be seen that the lowest standard deviation score is 47.57 , and the highest is 77.89 (this does not match the considerable variation of mean and median). Meanwhile, maximum skewness is a round 1.66 , with a minimum of about 0.29 showing all the distributed data is more leaning to the left. The level of tiles around the values most often arises by the parameters of kurtosis. All category images of people have a flatter (platykurtic) distribution than the normaldistribution, except those with a kurtosis score of about 5.22 .
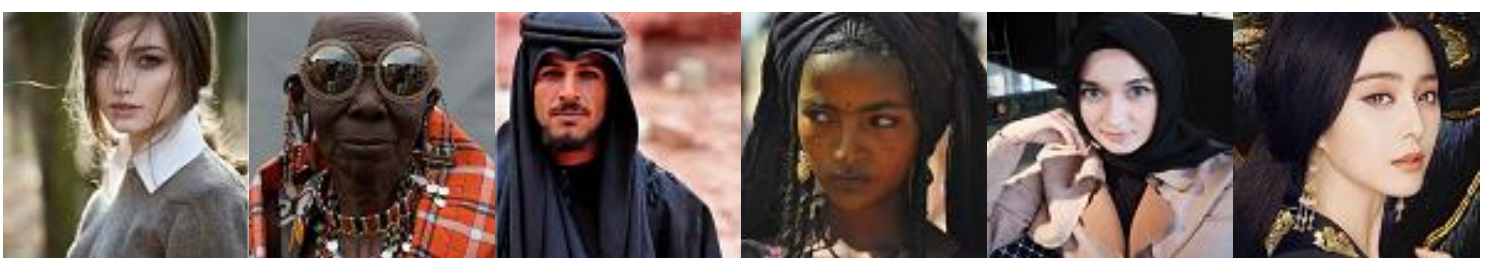

Figure 9. Six images for representing various people

Figure 10 shows some randomly chosen scenery category images without taking into account all types of images to represent the category [23]. The matrices contain numbers whose range is almost the maximum of 255 (listed in Table 2). Therefore, the numbers in the matrix do not show the typical properties of the specificness of the scenic view. Similarly, mean and median values, which tend to be les s consistent, indicating that the spread also varies throughout the range of values. It can also be seen that the lowest standard deviation score is 53.96, and the highest is 70.83 (this is not in line with the variations of the mean and median). Then, the maximum skewness value is about 0.61 with a minimum value of about -1.06 , indicating some of the data is distributed more leaning to the left, and some other data is more leaning to the right. The level of transient distribution around the most frequent values is shown by the parameters of kurtosis. All scenery category images have a flatter (platykurtic) distribution than the normaldistribution, except for scenary 6 with a kurtosis value slightly higher than 3 . 

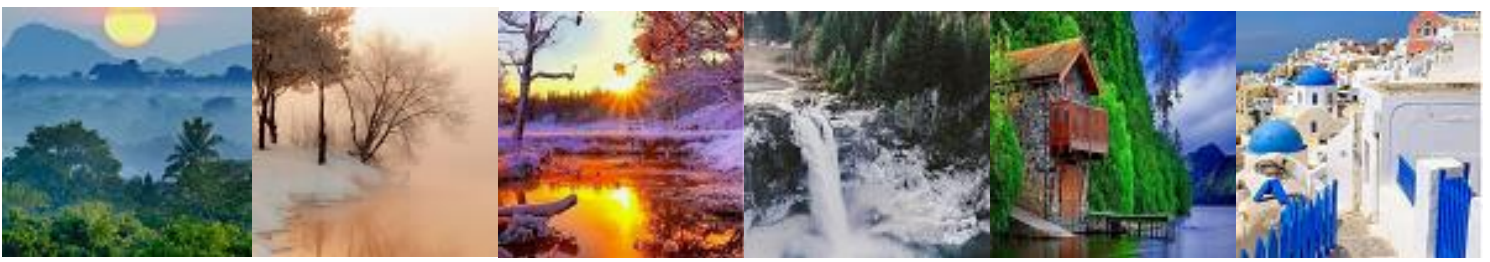

Figure 10. Six images for representing various scenery

Table 3 shows the average comparison of some statistical parameters between skin diseases and images from other categories. In the table, statistical parameter data from skin disease images in red color to facilitate the comparison process. In general, the parameter value of the skin disease image is different from the parameter value of the other categorical images. The skin disease image has the highest minimum value (29.5) and the lowest maximum value (225.5). So automatically, the image also has the smallest range. The mean value of the skin disease image is much greater than the mean of the other images. This is also in line with its larger median value (148.83).

Table 2. Properties of representational matrices of images of animal, architecture, art, vehicle, food, people,

\begin{tabular}{|c|c|c|c|c|c|c|c|c|}
\hline \\
\hline & Min & Max & Range & Mean & Median & Standard Deviation & Skewness & Kurtosis \\
\hline \multicolumn{9}{|l|}{ Animal } \\
\hline animall & 0 & 249 & 249 & 115.43 & 131 & 59.31 & -0.3202 & 2.0649 \\
\hline animal2 & 0 & 245 & 245 & 76.51 & 67 & 46.98 & 0.8602 & 3.2218 \\
\hline animal3 & 0 & 249 & 249 & 117.24 & 114 & 65.10 & 0.2723 & 2.1277 \\
\hline animal4 & 0 & 243 & 243 & 113.68 & 113 & 48.76 & -0.1461 & 1.9835 \\
\hline animal5 & 0 & 255 & 255 & 123.33 & 129 & 52.51 & -0.3991 & 2.5074 \\
\hline animal6 & 0 & 247 & 247 & 138.70 & 130 & 51.21 & 0.1693 & 2.0193 \\
\hline \multicolumn{9}{|l|}{ Architecture } \\
\hline architecturel & 0 & 255 & 255 & 89.55 & 57 & 75.34 & 0.3267 & 1.5250 \\
\hline architecture 2 & 0 & 255 & 255 & 107.21 & 98 & 65.98 & 0.4301 & 2.1913 \\
\hline architecture3 & 0 & 255 & 255 & 84.18 & 70 & 55.93 & 0.8594 & 3.0072 \\
\hline architecture 4 & 0 & 255 & 255 & 154.37 & 161 & 52.45 & -0.5957 & 2.8387 \\
\hline architecture 5 & 0 & 249 & 249 & 172.44 & 180 & 55.69 & -1.0032 & 3.2915 \\
\hline architecture6 & 0 & 255 & 255 & 125.73 & 124 & 62.24 & 0.0410 & 2.1777 \\
\hline \multicolumn{9}{|l|}{ Art } \\
\hline artl & 0 & 255 & 255 & 108.96 & 94 & 65.24 & 0.6053 & 2.3469 \\
\hline art2 & 25 & 255 & 230 & 97.91 & 89 & 33.59 & 1.2784 & 4.6723 \\
\hline $\operatorname{art3}$ & 0 & 255 & 255 & 112.75 & 111 & 42.53 & 0.1889 & 2.6732 \\
\hline art4 & 0 & 255 & 255 & 207.74 & 247 & 59.21 & -1.3847 & 3.8602 \\
\hline $\operatorname{art5}$ & 0 & 255 & 255 & 102.04 & 77 & 87.62 & 0.3329 & 1.5342 \\
\hline art6 & 0 & 249 & 255 & 149.68 & 155 & 35.44 & -0.6092 & 4.2599 \\
\hline \multicolumn{9}{|l|}{ Vehicle } \\
\hline vehiclel & 0 & 255 & 255 & 139.84 & 133 & 78.24 & -0.1269 & 1.4764 \\
\hline vehicle2 & 0 & 255 & 255 & 120.20 & 122 & 62.21 & -0.0445 & 1.8814 \\
\hline vehicle3 & 0 & 255 & 255 & 120.97 & 107 & 73.18 & 0.2029 & 1.6224 \\
\hline vehicle4 & 0 & 255 & 255 & 126.84 & 131 & 90.56 & -0.0966 & 1.4319 \\
\hline vehicle 5 & 0 & 255 & 255 & 94.30 & 85 & 74.64 & 0.7165 & 2.4487 \\
\hline vehicle6 & 0 & 255 & 255 & 78.69 & 71 & 58.55 & 0.9516 & 3.5275 \\
\hline \multicolumn{9}{|l|}{ Food } \\
\hline foodl & 0 & 255 & 255 & 124.56 & 133 & 74.48 & -0.1547 & 1.7741 \\
\hline food2 & 0 & 255 & 255 & 114.01 & 115 & 74.14 & 0.0435 & 1.7587 \\
\hline food 3 & 0 & 255 & 255 & 75.74 & 69 & 60.78 & 0.6186 & 2.5395 \\
\hline food4 & 0 & 255 & 255 & 132.00 & 138 & 67.17 & -0.1852 & 2.0898 \\
\hline food5 & 0 & 255 & 255 & 135.35 & 139 & 75.24 & -0.1102 & 1.6528 \\
\hline food6 & 0 & 255 & 255 & 78.22 & 68 & 58.26 & 0.7230 & 2.8148 \\
\hline \multicolumn{9}{|l|}{ People } \\
\hline peoplel & 0 & 255 & 255 & 113.00 & 102 & 57.61 & 0.4136 & 2.2457 \\
\hline people 2 & 0 & 255 & 255 & 86.91 & 80 & 57.54 & 0.3387 & 2.2824 \\
\hline people3 & 0 & 255 & 255 & 93.52 & 69 & 76.11 & 0.4990 & 1.8939 \\
\hline people 4 & 0 & 255 & 255 & 60.75 & 41 & 47.57 & 1.6627 & 5.2234 \\
\hline people 5 & 0 & 255 & 255 & 96.09 & 87 & 77.89 & 0.2968 & 1.5456 \\
\hline people6 & 0 & 255 & 255 & 84.40 & 44 & 76.03 & 0.7693 & 1.9838 \\
\hline \multicolumn{9}{|l|}{ Scenery } \\
\hline sceneryl & 0 & 251 & 251 & 105.44 & 96 & 55.91 & 0.1238 & 2.0897 \\
\hline scenery 2 & 0 & 255 & 255 & 159.58 & 165 & 53.96 & -0.4905 & 2.8618 \\
\hline scenery 3 & 0 & 255 & 255 & 124.37 & 117 & 70.83 & 0.1304 & 1.9448 \\
\hline scenery4 & 7 & 245 & 238 & 110.77 & 101 & 62.57 & 0.1796 & 1.5240 \\
\hline scenery5 & 0 & 250 & 250 & 85.42 & 77 & 58.20 & 0.6109 & 2.4908 \\
\hline scenery6 & 0 & 255 & 255 & 172.54 & 191 & 62.81 & -1.0627 & 3.4791 \\
\hline
\end{tabular}

Image investigation using higher moment statistics and edge detection for recognizing... (Afdhal Afdhal) 
Unlike the mean and median values, the standard deviation value of the skin disease image is the smallest compared to the mean values of the other images. This different illustrates that the difference in values in the matrix is not too significant to the average value. In other words, the possibility of edges on the image of skin disease is not too sharp. The average skewness values vary, but the variation is minimal, and almost all values are close to 0 . This variation indicates that the analyzed images are, on ave rage close to symmetry. Practically all matrices of the decomposed image are distributed more leaning to the left, except for the matrices of the image of the slightly leaning to the right (the average curve value is 3.2245). After knowing the characteristics of skin disease images based on statistical parameters, then next, we can design a unique processor minimalist just for image processing of skin diseases.

Table 3. Comparison of the average of the statistical parameter between skin disease and other images

\begin{tabular}{lcccccccc}
\hline & \multicolumn{7}{c}{ Average } \\
\cline { 2 - 8 } & Min & Max & Range & Mean & Median & Standard Deviation & Skewness & Kurtosis \\
\hline Skin & 29.5 & 255 & 225.50 & 155.37 & 148.83 & 47.8017 & 0.0108 \\
Animal & 0 & 248 & 248 & 114.15 & 114 & 53.9783 & 0.0727 & 2.3773 \\
Architecture & 0 & 254 & 254 & 122.25 & 115 & 61.2717 & 0.0097 & 2.5052 \\
Art & 4.17 & 254 & 249.83 & 129.85 & 128.83 & 53.9383 & 0.0686 & 3.2245 \\
Vehicle & 0 & 255 & 255 & 113.47 & 108.17 & 72.8967 & 0.2672 & 2.0647 \\
Food & 0 & 255 & 255 & 109.98 & 110.33 & 68.3450 & 0.1558 & 2.1050 \\
People & 0 & 253.67 & 253.67 & 89.11 & 70.50 & 65.4583 & 0.6634 & 2.5291 \\
Scenery & 1.17 & 251.83 & 250.67 & 126.35 & 124.50 & 60.7133 & -0.0848 & 2.3984 \\
\hline
\end{tabular}

\section{EDGE DETECTION OF SKIN IMAGES}

A block diagram of the system for identifying the abnormal skin based on the image is shown in Figure 11. Image 1 is an image of an allegedly ill (abnormal) skin, while Ima ge 2 is a normal image surrounding the supposedly abnormalskin. Both images will be inserted into the Image Classification section to determine whether included in the category of skin images or not. The classification process is based on the unique characteristics that distinguish the skin image from other drawings, as shown in Table 3.

Furthermore, both images are passed to the edge detection. Having done the edge detection process, the two images are compared to the level of similarity with each other. If both images are similar (high-similarity values), then it is decided that the skin whose image is analyzed is normal. Meanwhile, if the opposite, the value of similarity is low, then the skin may be abnormalor exposed to disea ses.

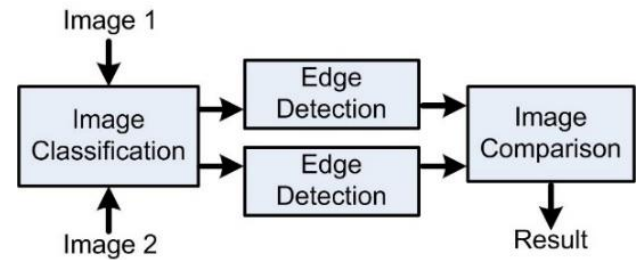

Figure 11. Block diagram of the skin disea se detection system through the image

To detect abnormalities in the skin through the image, several ways can be done. One of them is by applying an edge detection technique. This technique will process grayscale images in general to detect significant differences in intensity between adjacent pixels [25]. This technique is usually used for image segmentation and data extraction in the field of image processing, machine vision, and computer vision [26]. The edge detection technique involves a variety of mathematicalmethods that allaim to identify dots on digital images where the brightness of the image changes rapidly-the dots in the area where the image brightness changes drastically are usually arranged into a set of line curve segments called edges. Edge detection is an essential tool in image processing, even in the field of feature detection and extraction $[18,19]$.

Several algorithms are of ten used for edge detection, including Sobel, Canny, Prewitt, and fuzzy logic. Each of these algorithms has advantages and disadvantages [27]. In the paper, we used the Sobel technique for the edge detection of skin disease images. At this stage, we only apply the Sobel technique, leave it for future research for choosing the best technique of this application. The initial investigation of this technique using the Canny method was rea lized in the form of an Android-based application program [28]. However, the results 
of the analysis are still difficult to determine the abnormality of the skin. The images of skin disea ses as seen in Figure 1, will be in the edge detection process gradually to produce the last image used to determine a skin's abnormality. Then the results of the process will also be compared with the results of normal skin edge detection.

Referring to edge detection using the Sobel technique, several steps must be taken for analyzing the image. Those steps such as grayscaling image (step 1), detecting the whole cell (step 2), dilating the image (step 3), filling interior gaps (step 4), removing connected objects on the border (step 5), and smoothening the object (step 6). Figure 12 shows step by step edge detection with the Sobel technique on skin 1 of skin diseases [29].

To be able to do the edge detection process, the image must be two dimensional (2D) matrix. Therefore, the image of skin 1 in Figure 1 is changed to the grayscale color format, as shown in Figure 12(a). The second step is the process of detecting the whole cell with Sobel technique using fudge factor=0.75. The result of the first stage is shown in Figure 12(b). Figure 12(c) shows the results of the dilating process of the image using the strel function using the parameter 'line,' and the binary gradient mask process is widened using vertical and horizontalelementalstructures.

The next step process is filling interior gaps with the imfill function using parameter 'holes.' The results of this stage can be seen in Figure 12(d). Then, the following process is removing related objects and borders (border). The function used in this stage is imclearborder using clear level 4, and the results are shown in Figure 12(e). The final step is the process of refining the texture of the image with the strel function using the 'diamond' parameter. The result is, as shown in Figure 12(f).

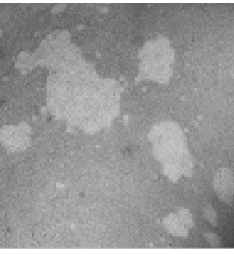

(a)

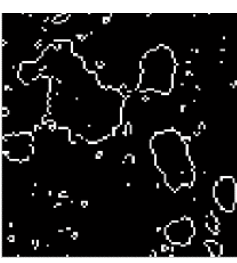

(b)

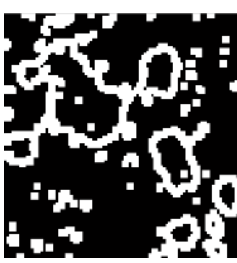

(c)

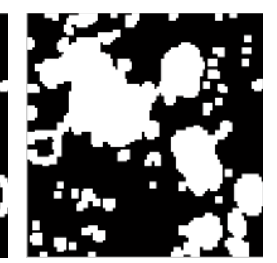

(d)

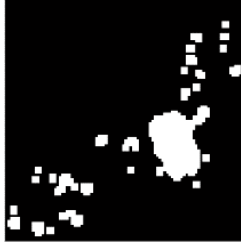

(e)

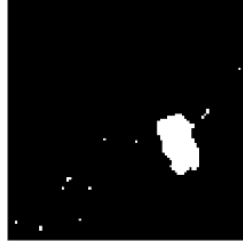

(f)

Figure 12. Edge detection steps of skin disease image of skin1, (a) grayscale image, (b) detecting entire cell, (c) dilating the image, (d) filling interior gaps, (e) removing connected objects on the border,

(f) smoothening the object

Referring to the edge detection results of some skin images, it is tough to know that the skin analyzed is not normal. Therefore it needs comparison with normal skin image. Figure 13 shows the correlation between skin6 and normal image on each step of the edge detection process. If it refers to the system design as in Figure 11, it is challenging to see the difference between the images of normal skin results from the abnormalskin. Therefore, the comparison will be evaluated even before the result of the last stage of the edge detection process.

In Figure 13, the process stage edge detection of skin6 (left side) and edge detection of normal skin (right side). Of all the stages, it is seen that the difference is very striking is the final stage (step 6). However, further analysis can be done by directly comparing the color in tensity of normal skin images with abnormal skin images at each stage. If there is no significant difference between both images at each stage, then it is necessary to examine the effect of changing the parameter on every step of the edge detection process.

In order to find the similarity between normal skin and skin6, we try to find the difference between them by subtracting the matrix of normal skin to the matrix of skin6 in every stage. The result of the subtraction is a new matrix containing entries positive or negative. The next step is taking the absolute value of entries to convert them to positive. After that, we calculate the mean (average) of the matrix and consider the results as the similarity value, as shown in Figure 13 on the last right collum.

The difference between normal and abnormal skins in stage $1($ mean=47.2017) is complicated to be distinguished because the color distribution is evenly distributed across the range of values from 0 to 255 . The different mean of stage 6 is very small, which is about 0.0229 . This number indicates that the image of normal skin and image of skin6 quite similar. Therefore, the image of skin6 indicates that the skin is maybe normal (no disease). However, as the figure was captured on the abnormal skin, the conclusion may be evaluated further to determine the suitable threshold (mean) in making a decision. 


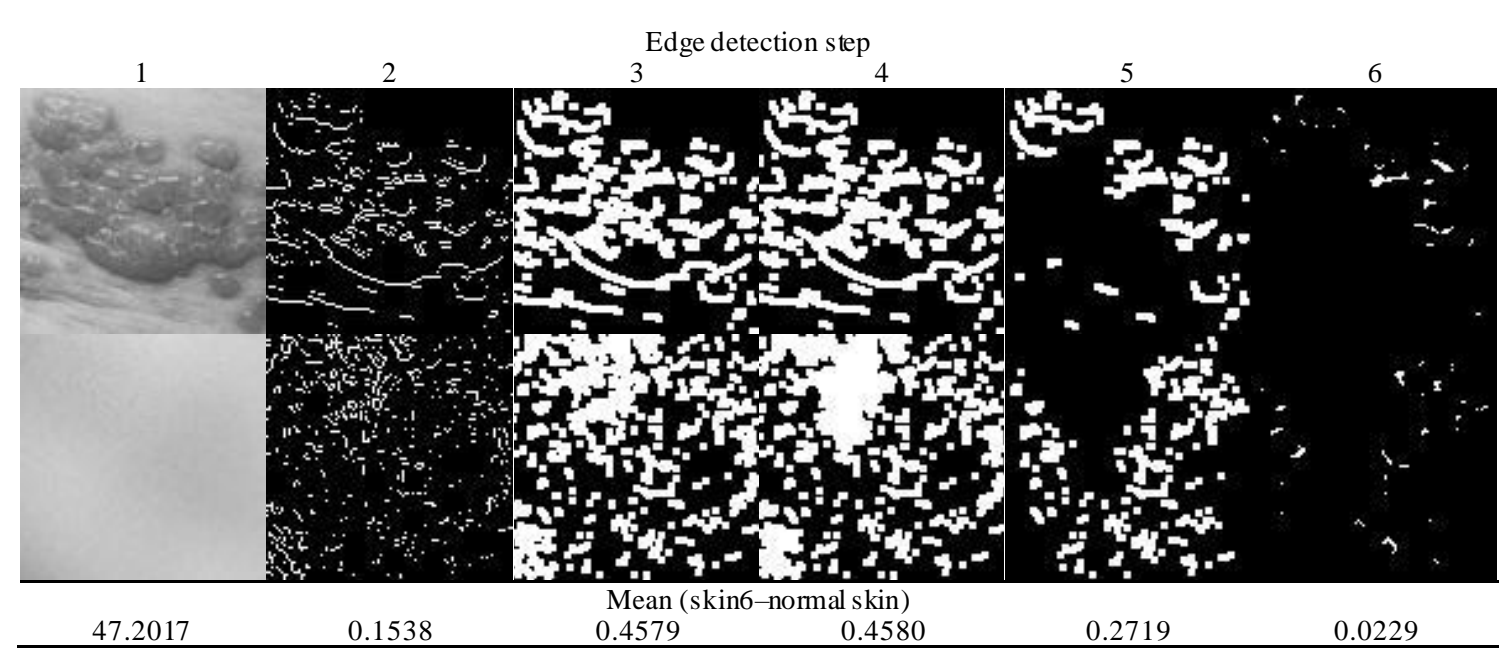

Figure 13. Comparison between edge detection process steps between skin 6 and normalskin

Another analysis can also be done on the comparison of the mean matrix of the two images in the second, third, fourth, and fifth stages. As seen in Figure 13, the mean differe nce between the skin6 image and the normal image is $0.1538,0.4579,0.4580$, and 0.2719 for the second, third, fourth, and fifth stages, respectively. In general, these values are greater than the comparison of the mean value in the sixth stage. However, if a comparison is required before the last stage, different threshold values must be determined for different stages [30].

\section{CONCLUSIONS}

Investigations using high-level statistical moments and edge detection for abnormal skin recognition have been done successfully. Six skin disea se images have been a nalyzed using several statistical parameters, including high-level moments such as skewness and kurtosis. For comparison, some images from other categories have been examined, as well. The results were compared with the results of the analysis of skin disease images. In general, the parametervalue of the skin disea se image is different from the parametervalue of the other categorical images. A system for detecting skin disease based on an image has been designed. The system also requires an edge detection process against the image of skin disease and normal skin. Sobel algorithm has been applied using MATLAB for detecting the edge. However, the results of the edge detection process, even in every stage, cannot distinguish a skin's abnormalities yet. Skin abnormalities may also be determined at stages before the final stage of the edge detection process. Therefore, it is necessary to adjust some of the selected parameters in several edge detection steps for more appropriate decision results.

\section{ACKNOWLEDGMENT}

This project is funded by the Universita s Syiah Kuala, the ministry of research, technology, and higher education, Indonesia under the scheme "Penelitian Strategis Nasional Institusi," No. 109/UN1 1.2/PP/SP3/2018, February 5, 2018.

\section{REFERENCES}

[1] J. Teevan, K. Collins-Thompson, R. W. White, S. T. Dumais, and Y. Kim, "Slow search: Information retrieval without time constraints," in Proceedings of the Symposium on Human-Computer Interaction and Information Retrieval, Canada, pp. 1-10, Oct 2013.

[2] B. Jiang and F. J. Ormeling, "Cybermap: the map for cyberspace," The Cartographic Journal, vol. 34, no. 2, pp. 111-116, 1997.

[3] K.-K. R. Choo, "The cyber threat landscape: Challenges and future research directions," Computers \& Security, vol. 30, no. 8, pp. 719-731, 2011.

[4] S. Weisgrau, "Issues in rural health: Access, hospitals, and reform," Health care financing review, vol. 17, no. 1, pp. 1-14, 1995 
[5] D. K. Silalahi, "Hubungan kebersihan perorangan dan pemakaian alat pelindung diri dengan keluhan gangguan kulit pada petugas pengelola sampah di tempat pembuangan akhir (tpa) namo bintang kecamatan pancur batu kabupaten deli serdang tahun 2010," Tesis, Public Health, Universitas Sumatera Utara, 2010.

[6] F. Efendi, M. Purwaningsih, A. Kurniati, and A. Bushy, "What do Indonesian nurses want? Retaining nurses in rural and remote areas of Indonesia," Online Journal of Rural Nursing and Health Care, vol. 14, no. 2, pp. 32-42, 2014.

[7] L. Chan, L. G. Hart, and D. C. Goodman, "Geographic access to health care for rural medicare beneficiaries," The Journal of Rural Health, vol. 22, no. 2, pp. 140-146, 2006.

[8] NIH MedlinePlus Magazine, "Skin health and skin diseases: Care for conditions from acne to wrinkles," Vitality Communications, 22-25, 2008. Available: https://medlineplus.gov/magazine/issues/fall08/articles/fall08pg2225.html.

[9] O. Kwan and J. Friel, "Clinical relevance of the sick role and secondary gain in the treatment of disability syndromes," Medical hypotheses, vol. 59, no. 2, pp. 129-134, 2002.

[10] R. Khattree and M. Bahuguna, "An alternative data analytic approach to measure the univariate and multivariate skewness," International Journal of Data Science and Analytics, vol. 7, pp. 1-16, 2019.

[11] A. D. Chave, "Computational statistics in the earth sciences: with applications in MATLAB," Cambridge University Press, 2017.

[12] S. Makino and C. M. Chan, "Skew and heavy-tail effects on firm performance," Strategic Management Journal, vol. 38, no. 8, pp. 1721-1740, 2017.

[13] G. Samoradnitsky, "Stable non-Gaussian random processes: stochastic models with infinite variance," Routledge, 2017.

[14] P. H. Westfall, "Kurtosis as peakedness, 1905-2014. RIP," The American Statistician, vol. 68, no. 3, pp. 191-195, 2014.

[15] T. F. Crack, "Kurtosis as peakedness: the phoenix from the ashes," 2019.

[16] J. C. Vallejo and M. A. Sanjuan, "Predictability," in Predictability of Chaotic Dynamics, ed: Springer, pp. 91-127, 2017.

[17] L. G. McAlevey and A. F. Stent, "Kurtosis: a forgotten moment," International Journal of Mathematical Education in Science and Technology, vol. 49, no. 1, pp. 120-130, 2018.

[18] J. R. Parker, "Algorithms for image processing and computer vision," John Wiley \& Sons, 2010.

[19] T. Sahoo and S. Pine, "Design and Simulation of SOBEL Edge Detection Using MATLAB Simulink," International Research Journal of Engineering and Technology(IRJET), vol. 3, no. 5, pp. 3069-3072, 2016.

[20] S. Dev, Y. H. Lee and S. Winkler, "Systematic study of color spaces and components for the segmentation of sky/cloud images," 2014 IEEE International Conference on Image Processing (ICIP), pp. 5102-5106, Paris, 2014.

[21] A. W. M. Smeulders, M. Worring, S. Santini, A. Gupta and R. Jain, "Content-based image retrieval at the end of the early years," in IEEE Transactions on Pattern Analysis and Machine Intelligence, vol. 22, no. 12, pp. 1349-1380, Dec. 2000.

[22] Z. Zulfikar and Z. Zulhelmi, "Statistical investigation of skin image for disease analyzing in rural area using Matlab," 2017 International Conference on Electrical Engineering and Informatics (ICELTICs), pp. 189-194, Banda Aceh, 2017.

[23] P. Team. (November 30). Available: https://id.pinterest.com/

[24] Z. Nadila. (November 30). Available: https://www.instagram.com/zsalsadil/?hl=en

[25] A. Koschan and M. Abidi, "Detection and classification of edges in color images," in IEEE Signal Processing Magazine, vol. 22, no. 1, pp. 64-73, Jan. 2005.

[26] M. Song and D. Civco, "Road extraction using SVM and image segmentation," Photogrammetric Engineering \& Remote Sensing, vol. 70, no. 12, pp. 1365-1371, 2004.

[27] A. Kushwah, K. Gupta, A. Agrawal, G. Jain, and G. Agrawal, "A Review: Comparative Study of Edge Detection Techniques," International Journal of Advanced Research in Computer Science, vol. 8, no.5, pp. 2528-2531, 2017.

[28] Z. Zulfikar, Z. Zulhelmi, T. Y. Arif, A. Afdhal and P. N. Syawaldi, "Android Application: Skin Abnormality Analysis based on Edge Detection Technique," 2018 International Conference on Electrical Engineering and Informatics (ICELTICs), pp. 89-94, Banda Aceh, 2018.

[29] Wenshuo Gao, Xiaoguang Zhang, Lei Yang and Huizhong Liu, "An improved Sobel edge detection," $20103 r d$ International Conference on Computer Science and Information Technology, Chengdu, 2010, pp. 67-71.

[30] H. Zhang, Q. Zhu and X. Guan, "Probe into Image Segmentation Based on Sobel Operator and Maximum Entropy Algorithm," 2012 International Conference on Computer Science and Service System, Nanjing, 2012, pp. 238-241. 


\section{BIOGRAPHIES OF AUTHORS}
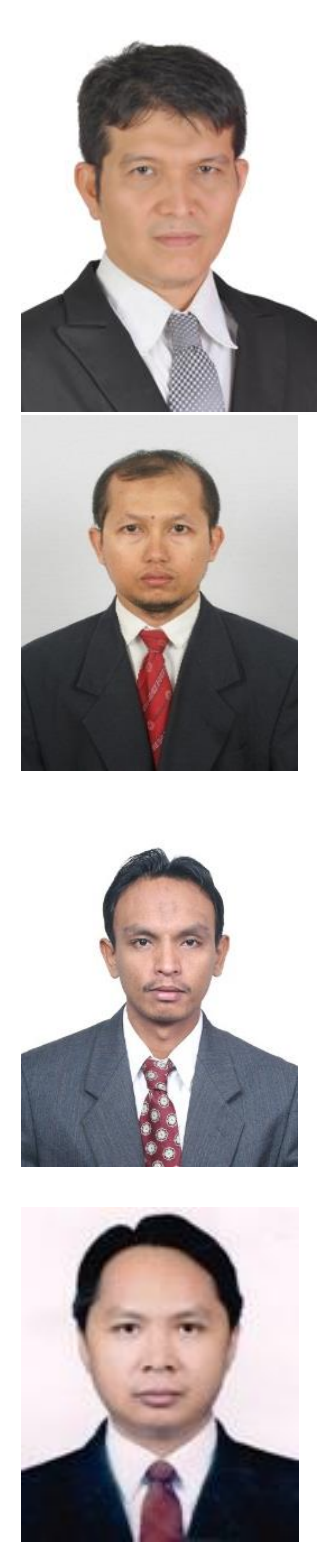

Afdhal (Afdhal Azmi) was born in Bireuen, Aceh, Indonesia, in 1979. He received a bachelor's degree in Computer System Engineering from the Department of Electrical Engineering, Universitas Syiah Kuala, Indonesia, in 2003. He finished master degree (M.Sc.) from School of Computer Sciences, Universiti Sains Malaysia, Malaysia, in 2013. He started working as a lecturer and researcher at the Department of Electrical and Computer Engineering, Universitas Syiah Kuala, from 2004 until the present. His research interests include Intelligent Transportation System, Vehicular Communication, Data Communication and Networking, Cooperative Networks, Ad-hoc Networks, Networks Security, Parallel and Distributed Systems, Internet of Things (IoT), Internet of Vehicles (IoV), Image and Pattern Recognition, and Multimedia Processing. Afdhal is a professional member of the Association for Computing Machinery (ACM), IEEE, IEEE SMC Society, and Internet Society (ISOC).

Zulfikar was born in Beureunuen, Aceh, Indonesia, in 1975. He received his B.E. degree in Electrical Engineering from North Sumatera University, Medan, Indonesia, and the M. Sc. Degree from King Saud University, Riyadh, Saudi Arabia, in 1999 and 2011, respectively. Currently, he is studying the Ph.D. program at the University of Malaya, Malaysia. He joined as teaching staff in the Department of Electronics at Politeknik Caltex Riau, Pekanbaru, Indonesia, in 2003. He served as head of Industrial Control Laboratory, Politeknik Caltex Riau, from 2003 to 2006. In 2006, he joined the Electrical Engineering Department, Syiah Kuala University. He has been appointed as head of Digital Laboratory for two successive years. His current research interests include VLSI design, System on Chips (SoC), and System for gathering renewable energy.

Zulhelmi was born in 1979 in Gigieng Simpang Tiga, which is located in Pidie District Aceh. He received a B.E. degree from Syiah Kuala University, Banda Aceh, Indonesia in 2003, and the M.Sc degrees in electrical engineering from King Saud University, Riyadh, Saudi Arabia in 2013. He has been a Lecturer at Electrical Engineering Department, Syiah Kuala University, since 2003. His research interests include Electronic design, FPGAs architecture design, microcontroller, microprocessor, sensor devices, biomedical sensing, and instrumentation systems. His recent publication is about empowering and the use of alternative energy, such as solar energy, to be utilized at citizen houses.

Teuku Yuliar Arif received the B.E. degree from Sepuluh Nopember Institute of Technology (ITS), Surabaya, Indonesia, in 1998, and the M.Com., and Ph.D. degrees from University of Indonesia (UI), Indonesia, in 2004 and 2013 respectively. Since 1999, he joined Syiah Kuala University, Banda Aceh, Indonesia, as a lecturer at the Electrical Engineering Department. His research interests include wireless and mobile networks, vehicular ad hoc networks (VANET), network security, network performance analysis, and mesh network for the Internet of Things (IoT). 